Supporting Information

\title{
Conformationally Flexible Sites within the Transmembrane Helices of Amyloid Precursor Protein and Notchı Receptor
}

Walter Stelzer and Dieter Langosch*

Lehrstuhl Chemie der Biopolymere, Technische Universität München, Weihenstephaner Berg 3, 85354 Freising, and Munich Center For Integrated Protein Science (CIPSM), Germany.

* corresponding author

\section{Contents:}

Materials and Methods

Supporting Table $S 1$

Supporting Figures $S_{1}, S_{2}, S_{3}$ and $S_{4}$

Supporting References 


\section{Materials and Methods}

Peptide design and synthesis. The TMD sequence Notch1 L1734 to S1757 was tagged with an $\mathrm{N}$-terminal KKK motif. We have previously found that a KKK motif facilitates fragmentation of the C99-derived A28-55 TMD peptide in the gas phase by the ETD method, which is required for determining site-specific exchange rate constants; yet it has only minimal impact on $\mathrm{D} / \mathrm{H}$ exchange ${ }^{1}$. The C-terminal RKR motif of the Notchı TMD was also replaced by KKK that delimits the C99 TMD. We deemed it essential to equip both TMDs with identical basic tags, that attract water, for a valid comparison of $\mathrm{D} / \mathrm{H}$ exchange at the adjacent hydrophobic sequences. Both peptides were capped at both termini to eliminate charges at the backbone. Peptides were synthesized by Fmoc chemistry (PSL, Heidelberg, Germany) and purified by HPLC. They were > 90\% pure as judged by mass spectrometry. Concentrations were determined via UV spectroscopy using an extinction coefficient at $205 \mathrm{~nm}$ of $128,820 \mathrm{M}^{-1} \cdot \mathrm{cm}^{-1}$ and $84,280 \mathrm{M}^{-1} \cdot \mathrm{cm}^{-1}$ respectively for Notchı and C99 peptide ${ }^{2}$.

Circular dichroism spectroscopy. For CD spectroscopy, peptides were dissolved at $50 \mu \mathrm{M}$ in $80 \%(\mathrm{v} / \mathrm{v})$ TFE in $5 \mathrm{mM}$ PBS, pH 7.4. CD spectra were obtained using a Jasco J-710 CD spectrometer from $190 \mathrm{~nm}$ to $260 \mathrm{~nm}$ in a $1.0 \mathrm{~mm}$ quartz cuvette at $20^{\circ} \mathrm{C}$ with a response of $1 \mathrm{~s}$, a scan speed of $100 \mathrm{~nm} / \mathrm{min}$ and a sensitivity of $100 \mathrm{mdeg} / \mathrm{cm}$. Spectra were the signal-averaged accumulations of 10 scans with the baselines (corresponding to solvent) subtracted and mean molar ellipticities were calculated.

Mass spectrometry and DHX experiments. Peptides were dissolved in $80 \%(v / v) d_{1^{-}}$ $\mathrm{HFIP} / 20 \% \mathrm{D}_{2} \mathrm{O}$ at a concentration of $300 \mu \mathrm{M}$ and incubated at $37^{\circ} \mathrm{C}$ for 7 days, solvent was removed by vacuum centrifugation, and the pellet was re-dissolved in deuterated solvent $(80 \%$ $(\mathrm{v} / \mathrm{v}) \mathrm{d}_{1}$-TFE, $2 \mathrm{mM} \mathrm{ND}_{4}$-acetate). This resulted in $>95 \%$ deuteration as determined by ESI-MS. Deuterated DTT was prepared by repeatedly dissolving DTT in $\mathrm{D}_{2} \mathrm{O}$ and lyophilizing overnight; the deuterated DTT was finally dissolved in $\mathrm{D}_{2} \mathrm{O}$. Deuterated DTT was added at $1 \mathrm{mM}$ to prevent cysteine oxidation in the deuterated peptides. For measurements of overall DHX, the deuterated peptides were diluted 1:20 from a $100 \mu \mathrm{M}$ stock solution into $80 \%(\mathrm{v} / \mathrm{v}) \mathrm{TFE}, 2 \mathrm{mM} \mathrm{NH}_{4}$-acetate, $1 \mathrm{mM}$ tris(2-carboxyethyl)phosphine (TCEP), $\mathrm{pH}$ 4.o, and incubated for different time periods at $20.0^{\circ} \mathrm{C}$ after which DHX was quenched on ice and by adding (o.5\% (v/v) formic acid (final $\mathrm{pH}$ $\approx 2.5$ ). Calculation of global DHX was done as described in ${ }^{4}$. For electron transfer dissociation (ETD), $300 \mu \mathrm{M}$ stock solutions were diluted 1:20. Mass/charge $(\mathrm{m} / \mathrm{z})$ ratios were recorded after the indicated time periods by electron-spray-ionization mass spectrometry (ESI-MS) as described ${ }^{3}$ using a Synapt G2 HDMS (Waters Co.) with one scan/second and evaluated as described ${ }^{4}$. For ETD-DHX, incubation times from 1 min to 3 days at $\mathrm{pH} 4.0$ were chosen. ETD was conducted as described ${ }^{5}$ at a flow rate of $3 \mu \mathrm{l} / \mathrm{min}$ using a cooled syringe. ETD was started by decreasing the wave height from $1.5 \mathrm{~V}$ to $0.2 \mathrm{~V}$. Hydrogen scrambling was $<5-10 \%$, as determined by the ammonia loss method ${ }^{6}$ with an unblocked referenc peptide. All experiments were done at least in triplicate. ETD fragment spectra recorded over 10 min were combined and 
evaluated in terms of site-specific $\mathrm{D} / \mathrm{H}$ exchange rate constants and free energies of $\mathrm{H}$-bond formation using the module ETD Fragment Analyzer of the software suite MassMap (MassMap GmbH \& Co. KG, Freising, Germany) that is based on GRAMS/AI (Thermo Fisher Scientific). Details are described in ${ }^{7}$. As in our previous publications 5,7 , we note that the calculation of equilibrium constants was done for a standard situation of exchange in pure water. The absolute values of $\mathrm{K}_{\mathrm{eq}}$ and $\Delta \mathrm{G}$ determined in $80 \%$ TFE may thus be influenced by a number of factors 5 . However, these factors influence $\Delta \mathrm{G}$ values of Notch 1 and $C_{99}$ peptides in a similar fashion, thus allowing their comparison.

Quantitative evaluation of enzyme/substrate structures. Helix dihedral angles were determined using the STRIDE webserver (http://webclu.bio.wzw.tum.de/cgibin/stride/stridecgi.py $)^{8}$. Rise per residue along the helix axis was determined using the HELANAL plus server where this metric is denoted by "height" (http://nucleix.mbu.iisc.ac.in/helanalplus/index.html) 9. Helical regions were defined as ranging from I31 to $\mathrm{I}_{47}(\mathrm{C} 83)$ and from $\mathrm{Al}_{1732}$ to $\mathrm{C}_{1752}$ (Notch1). 
Table S1Site-specific D/H exchange rate constants and free energies of helix formation

\begin{tabular}{|c|c|c|c|c|c|}
\hline Notch1 $^{1}$ & & & $\overline{C 99^{1}}$ & & \\
\hline$\frac{\frac{d}{\sigma}}{\frac{\delta}{\pi}}$ & $\begin{array}{l}\log \mathbf{k}_{\exp }{ }^{2} \\
{\left[\mathrm{~min}^{-1}\right]}\end{array}$ & $\begin{array}{l}\Delta \mathbf{G}^{3} \\
{[\mathrm{kcal} / \mathrm{mol}]}\end{array}$ & 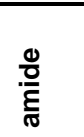 & $\begin{array}{l}\log k_{\exp } \\
{\left[\mathrm{min}^{-1}\right]}\end{array}$ & $\begin{array}{l}\Delta \mathbf{G} \\
{[\mathrm{kcal} / \mathrm{mol}]}\end{array}$ \\
\hline $\mathbf{K}$ & n.d. ${ }^{4}$ & n.d. & K & n.d. & n.d. \\
\hline K & n.d. & n.d. & K 28 & n.d. & n.d. \\
\hline L 1734 & n.d. & n.d. & G 29 & n.d. & n.d. \\
\hline H 1735 & n.d. & n.d. & A 30 & n.d. & n.d. \\
\hline F 1736 & n.d. & n.d. & I 31 & n.d. & n.d. \\
\hline M 1737 & n.d. & n.d. & 132 & $-0.98 \pm 0.03$ & n.d. \\
\hline Y 1738 & $-0.76 \pm 0.094$ & $0.36 \pm 0.2$ & G 33 & $-0.98 \pm 0.08$ & $1.0 \pm 0.1$ \\
\hline V 1739 & $-1.64 \pm 0.060$ & $1.0 \pm 0.1$ & L 34 & $-1.05 \pm 0.06$ & $0.34 \pm 0.1$ \\
\hline A 1740 & $-1.71 \pm 0.043$ & $1.9 \pm 0.06$ & M 35 & $-1.19 \pm 0.05$ & $0.93 \pm 0.1$ \\
\hline A 1741 & $-1.98 \pm 0.048$ & $2.4 \pm 0.06$ & V 36 & $-1.75 \pm 0.08$ & $1.2 \pm 0.1$ \\
\hline A 1742 & $-2.19 \pm 0.055$ & $2.7 \pm 0.1$ & G 37 & $-2.02 \pm 0.09$ & $2.6 \pm 0.1$ \\
\hline A 1743 & $-2.95 \pm 0.16$ & $3.7 \pm 0.2$ & G 38 & $-2.44 \pm 0.08$ & $3.6 \pm 0.1$ \\
\hline F 1744 & $-3.19 \pm 0.11$ & $3.8 \pm 0.2$ & V 39 & $-2.49 \pm 0.11$ & $2.4 \pm 0.1$ \\
\hline V 1745 & $-3.38 \pm 0.16$ & $3.5 \pm 0.3$ & V 40 & $-2.87 \pm 0.13$ & $2.5 \pm 0.2$ \\
\hline L 1746 & $-3.09 \pm 0.14$ & $3.0 \pm 0.2$ & I 41 & $-3.00 \pm 0.14$ & $2.6 \pm 0.2$ \\
\hline L 1747 & $-3.09 \pm 0.22$ & $2.9 \pm 0.3$ & A 42 & $-3.19 \pm 0.11$ & $3.7 \pm 0.2$ \\
\hline F 1748 & $-2.95 \pm 0.48$ & $3.2 \pm 0.7$ & $\mathrm{~T} 43$ & $-3.11 \pm 0.08$ & $3.8 \pm 0.1$ \\
\hline F 1749 & $-3.09 \pm 0.19$ & $3.7 \pm 0.3$ & V 44 & $-4.31 \pm 0.13$ & $4.8 \pm 0.2$ \\
\hline V 1750 & n.d. & n.d. & 145 & $-4.97 \pm 0.10$ & $5.2 \pm 0.2$ \\
\hline G 1751 & n.d. & n.d. & V 46 & $-4.97 \pm 0.11$ & $5.2 \pm 0.2$ \\
\hline C 1752 & n.d. & n.d. & I 47 & $-4.86 \pm 0.14$ & $5.1 \pm 0.2$ \\
\hline G 1753 & $-1.98 \pm 0.15$ & $3.2 \pm 0.2$ & T 48 & $-4.56 \pm 0.26$ & $5.4 \pm 0.4$ \\
\hline V 1754 & $-2.61 \pm 0.09$ & $2.6 \pm 0.1$ & L 49 & $-4.40 \pm 0.27$ & $5.1 \pm 0.4$ \\
\hline L 1755 & $-1.98 \pm 0.12$ & $1.5 \pm 0.2$ & V 50 & $-4.04 \pm 0.17$ & $3.9 \pm 0.2$ \\
\hline L 1756 & $-1.68 \pm 0.24$ & $0.91 \pm 0.4$ & M 51 & $-3.42 \pm 0.19$ & $4.1 \pm 0.3$ \\
\hline S 1757 & $-1.25 \pm 0.27$ & $1.6 \pm 0.4$ & L 52 & $-3.61 \pm 0.09$ & $3.9 \pm 0.1$ \\
\hline $\mathrm{K}$ & n.d. & n.d. & K 53 & n.d. & n.d. \\
\hline K & n.d. & n.d. & K 54 & n.d. & n.d. \\
\hline K & n.d. & n.d. & K 55 & n.d. & n.d. \\
\hline
\end{tabular}

${ }^{1}$ Identity of the TMD peptide

${ }^{2} \mathrm{D} / \mathrm{H}$ exchange rate constant $\mathrm{k}_{\mathrm{exp}}$ from exponential fit of means $\pm \mathrm{SEM}$

${ }^{3}$ free energy $\Delta G$ with confidence interval

${ }^{4}$ n.d. $=$ not determined 


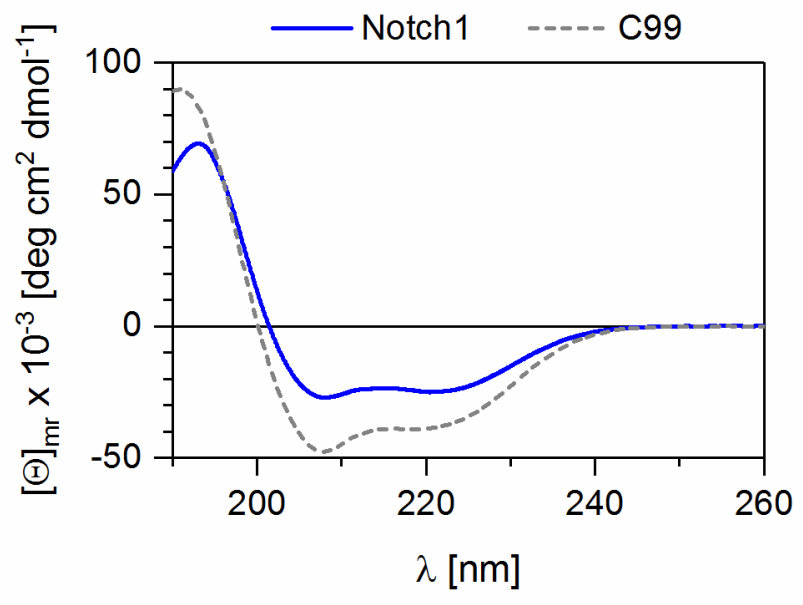

Figure S1 Secondary structure of Notchi and C99 TMD peptides determined by circular dichroism spectroscopy. Spectra were recorded in 80\% TFE, 5 mM PBS buffer (averaged spectra, $\mathrm{n}=3)$. 

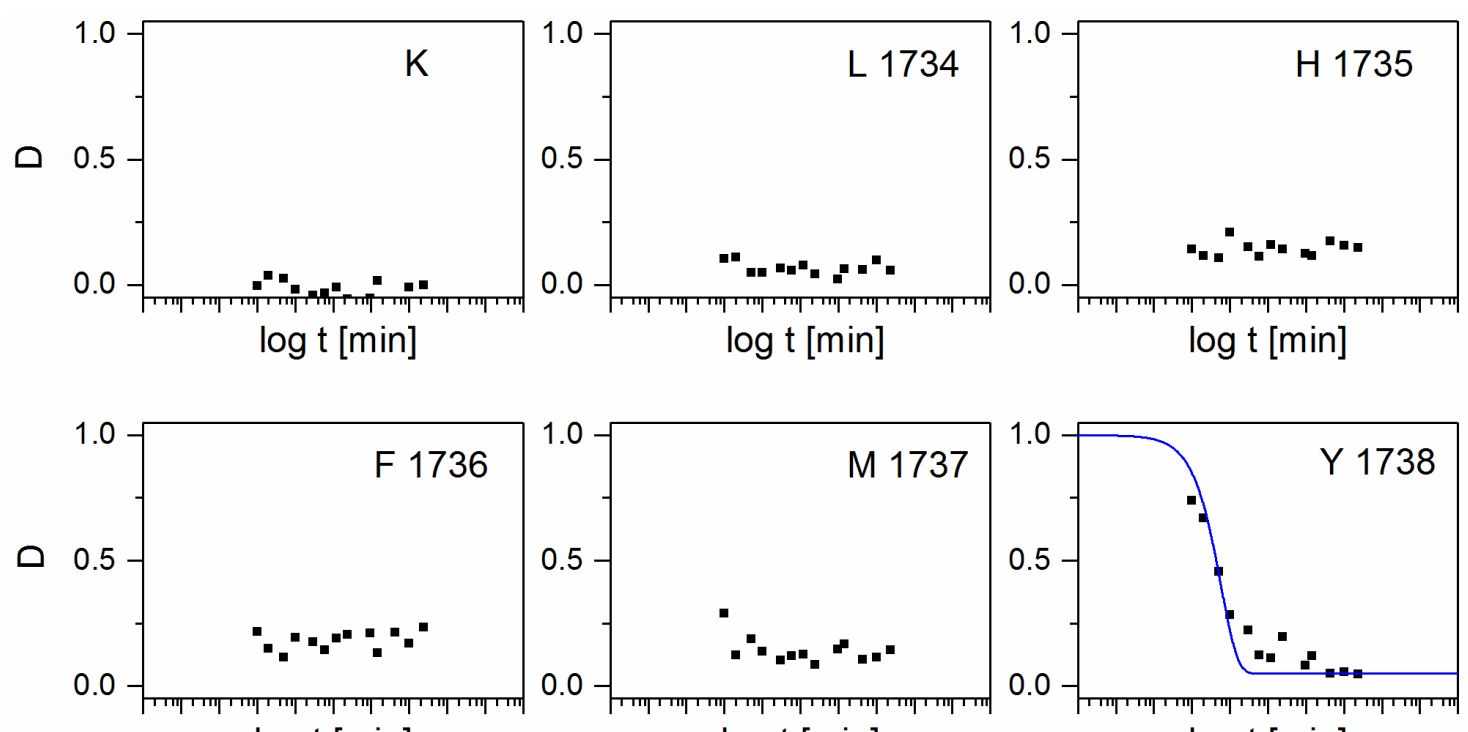

$\log \mathrm{t}[\mathrm{min}]$

$\log \mathrm{t}[\mathrm{min}]$

$\log \mathrm{t}[\mathrm{min}]$
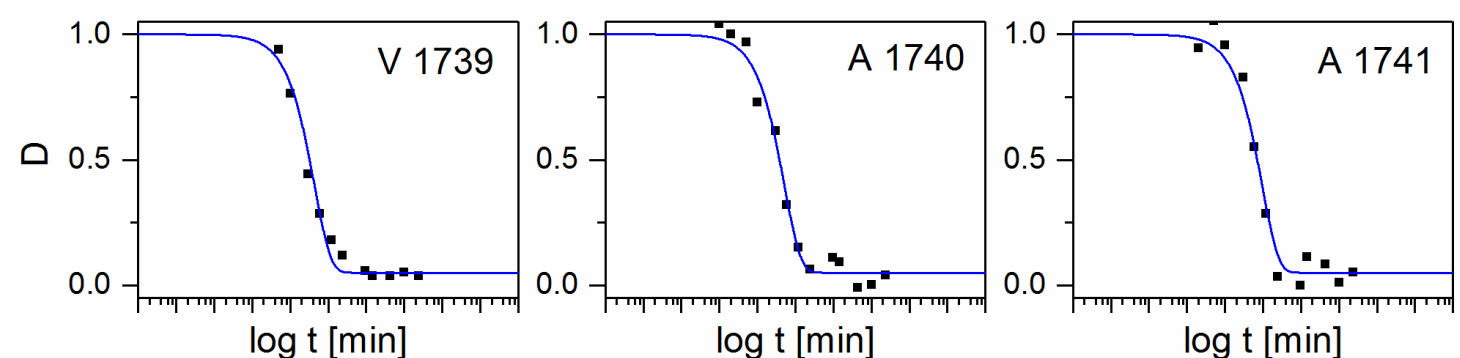

$\log \mathrm{t}[\mathrm{min}]$

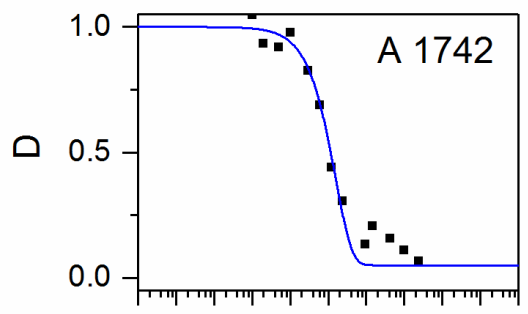

$\log \mathrm{t}[\mathrm{min}]$

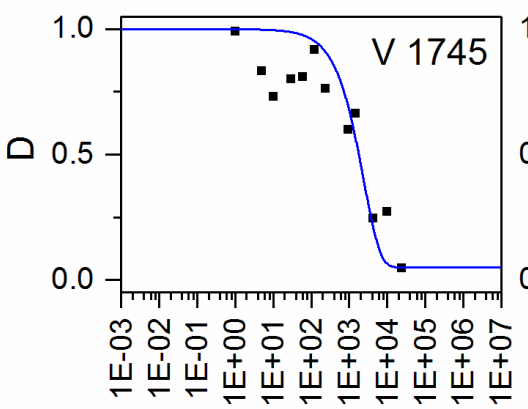

$\log t[\mathrm{~min}]$

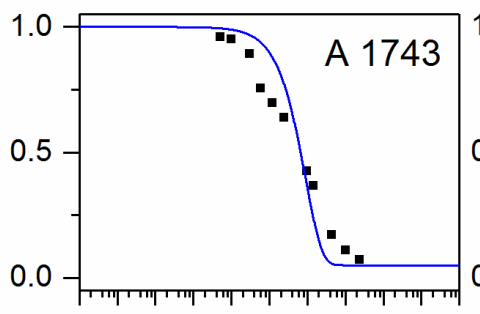

$\log \mathrm{t}[\mathrm{min}]$

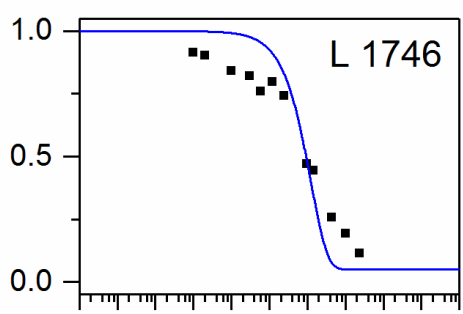

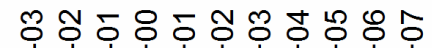

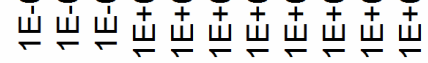

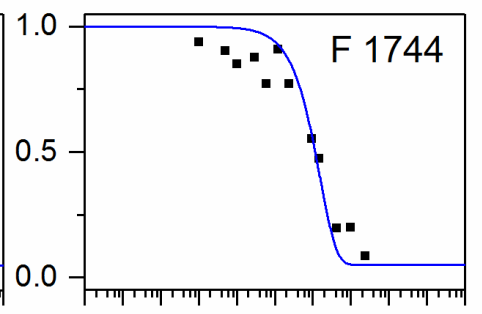

$\log t$ [min]

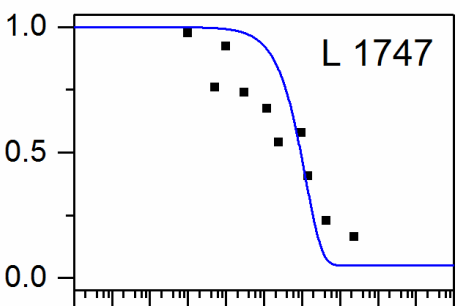

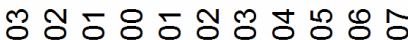

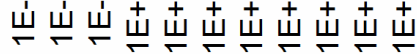

$\log t$ [min]

$\log t$ [min] 

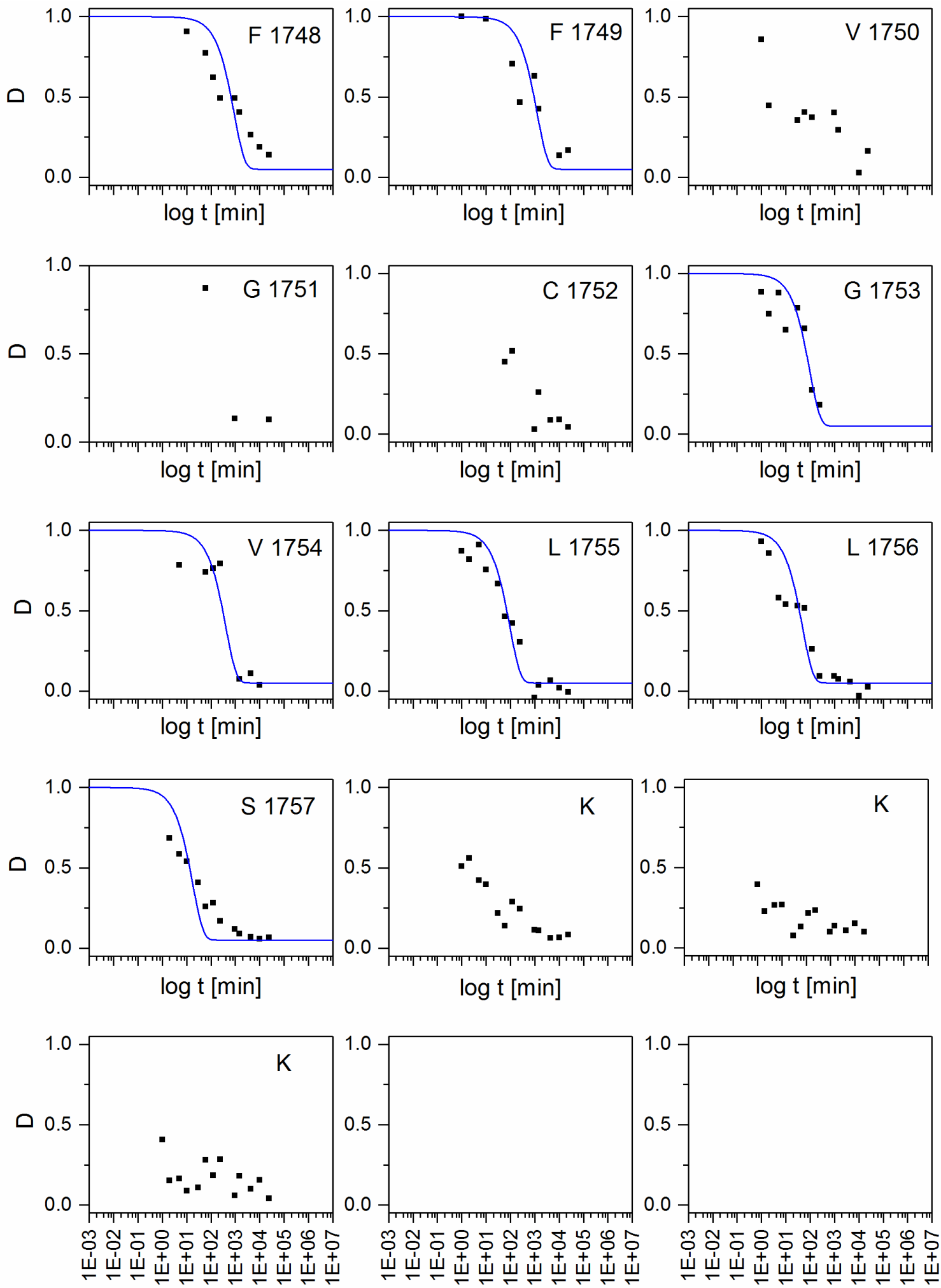

$\log \mathrm{t}[\mathrm{min}]$

$\log \mathrm{t}[\mathrm{min}]$

$\log t[\mathrm{~min}]$

Figure S2 Residue-specific D/H exchange kinetics of the Notch TMD peptide obtained after ETD. The calculated deuterium contents $D$ (mean values, $n \geq 3$ ) of the respective amides are plotted against the log of the exchange period t. The identity of the TMD residue is given in the insets. Exponential fits are depicted for those kinetics that were deemed complete enough for calculating the exchange rate constants given in Figure $2 \mathrm{~A}$ and in Table S1. 
presenilin-1

nicastrin

PEN-2

APH-1A

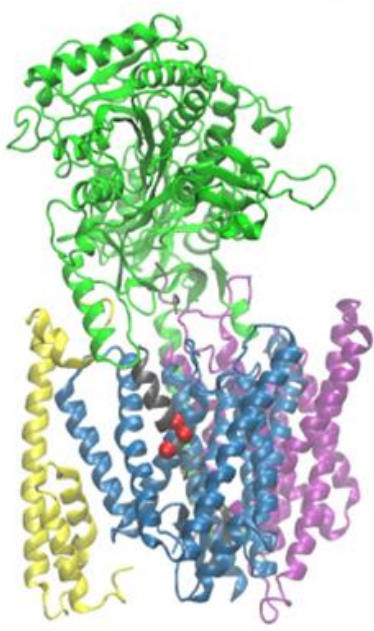

C83

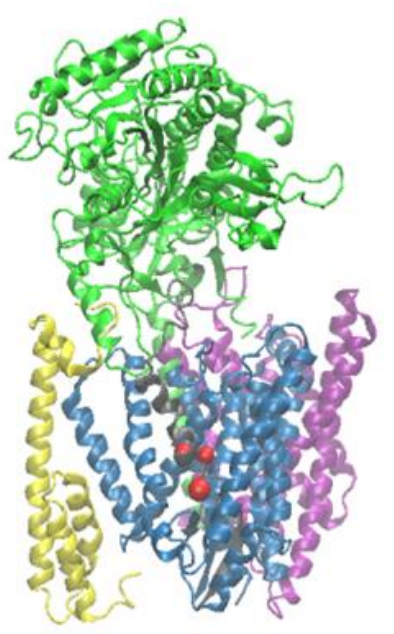

Notch1

Figure $\mathrm{S}_{3}$ Visualization of enzyme-bound $\mathrm{C}_{3} 3$ and Notch1 (in grey) within the structure of $\gamma$ secretase (left panel: with C83, pdb: 6IYC, ref. ${ }^{10}$; right panel: with Notch1, 6IDF, ref. ${ }^{11}$ ) using VMD ${ }^{12}$. Red spheres denote those residues shown here to form low-stability amide H-bonds (C83: V39, V40, I41; Notch1: L1746, L1747, F1748). 

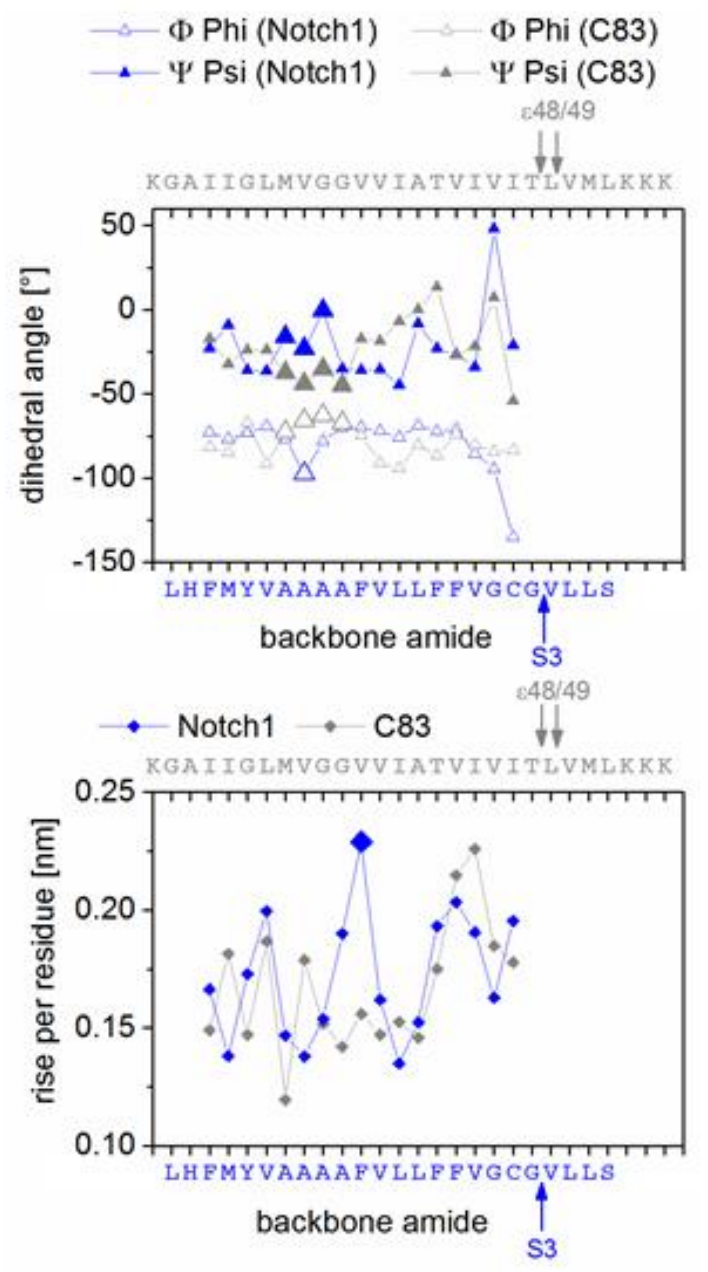

Figure $\mathbf{S}_{4}$ Structural parameters of substrate TMD helices within the respective enzyme/substrate complexes (C83, pdb: 6IYC; Notch1, 6IDF). A) Helix dihedral angles. B) Riseper-residue. Enlarged symbols denote residues near the putative hinge sites where values appear to deviate from values of neighboring amino acids. We note that these trends must be viewed with caution, given the limited resolution of the structures $(0.26 \mathrm{~nm}$ to $0.27 \mathrm{~nm})$. 


\section{Supporting References}

1. Gotz, A.; Hogel, P.; Silber, M.; Chaitoglou, I.; Luy, B.; Muhle-Goll, C.; Scharnagl, C.; Langosch, D., Increased H-Bond Stability Relates to Altered epsilon-Cleavage Efficiency and Abeta Levels in the 145T Familial Alzheimer's Disease Mutant of APP. Sci Rep 2019, 9 (1), 5321.

2. Anthis, N. J.; Clore, G. M., Sequence-specific determination of protein and peptide concentrations by absorbance at $205 \mathrm{~nm}$. Protein Sci 2013, 22 (6), 851-8.

3. Poschner, B. C.; Quint, S.; Hofmann, M.; Langosch, D., Sequence-specific conformational dynamics of model transmembrane domains determines their membrane fusogenic function. J. Mol. Biol. 2009, 386, 733-741.

4. Stelzer, W.; Poschner, B. C.; Stalz, H.; Heck, A. J.; Langosch, D., Sequence-specific conformational flexibility of SNARE transmembrane helices probed by hydrogen/deuterium exchange. Biophys. J. 2008, 95, 1326-1330.

5. Stelzer, W.; Scharnag, C.; Leurs, U.; Rand, K. D.; Langosch, D., The impact of the 'Austrian' mutation of the amyloid precursor protein transmembrane helix is communicated to the hinge region. Chemistry Select 2016, 1, 4408-4412.

6. $\quad$ Rand, K. D.; Pringle, S. D.; Morris, M.; Brown, J. M., Site-Specific Analysis of Gas-Phase Hydrogen/Deuterium Exchange of Peptides and Proteins by Electron Transfer Dissociation. Analytical Chemistry 2012, 84 (4), 1931-1940.

7. Yucel, S. S.; Stelzer, W.; Lorenzoni, A.; Wozny, M.; Langosch, D.; Lemberg, M. K., The Metastable XBP1u Transmembrane Domain Defines Determinants for Intramembrane Proteolysis by Signal Peptide Peptidase. Cell Reports 2019, 26 (11), 3087 - 3099.

8. Frishman, D.; Argos, P., Knowledge-based protein secondary structure assignment. Proteins 1995, 23 (4), 566-79.

9. Kumar, P.; Bansal, M., HELANAL-Plus: a web server for analysis of helix geometry in protein structures. J Biomol Struct Dyn 2012, 30 (6), 773-83.

10. Zhou, R.; Yang, G.; Guo, X.; Zhou, Q.; Lei, J.; Shi, Y., Recognition of the amyloid precursor protein by human gamma-secretase. Science 2019.

11. Yang, G.; Zhou, R.; Zhou, Q.; Guo, X.; Yan, C.; Ke, M.; Lei, J.; Shi, Y., Structural basis of Notch recognition by human $\psi$-secretase. Nature 2019, 565 (7738), 192-197.

12. Humphrey, W.; Dalke, A.; Schulten, K., VMD: visual molecular dynamics. Journal of molecular graphics 1996, 14 (1), 33-8, 27-8. 